\title{
Seven years of experience with zygomycosis in Iran: a seasonal disease
}

\section{Authors}

Ali Zare Mirzaie ${ }^{1}$

Akram Jahangiri $^{2}$

Alireza Sadeghipour ${ }^{3}$

Nasrin Shayanfar ${ }^{1}$

${ }^{1} \mathrm{MD}$, Assistant Professors of Pathology, Tehran University of Medical Sciences and Health Services, Tehran, Iran ${ }^{2} \mathrm{MD}$, General Practitioner, Tehran University of Medical Sciences and Health Services, Tehran, Iran

${ }^{3} \mathrm{MD}$, Associate Professor of Pathology, Tehran University of Medical Sciences and Health Services, Tehran, Iran

Submitted on: 03/30/2011 Approved on: 04/03/2011

Correspondence to: Ali Zare Mirzaie Tehran University of Medical Sciences and Health Services

Department of Pathology Rasool Akram Medical Complex Sattarkhan-Niayesh St. 14455- 364, Tehran, Iran zaremir@gmail.com

We declare no conflict of interest.

(C2011 Elsevier Editora Ltda. All rights reserved.
Dear Editor,

Zygomycosis, earlier considered as rare entity, is being reported with increasing frequency in recent years. ${ }^{1}$ Classically described predisposing factors include poorly controlled diabetes, especially when associated with ketoacidosis, corticosteroid use, immunosuppression therapy for solid organ transplant or bone marrow transplant, neutropenia or neutrophil dysfunction associated with leukemia/lymphoma. ${ }^{2,3}$ In one study in Lebanon, it has been reported that mucormycosis incidence appears to be seasonal in the Eastern Mediterranean and clustering of onset of invasive mucormycosis begins around May and ends in October. ${ }^{4}$ In order to understand the whole gamut of the disease in the Iranian scenario, we undertook a retrospective analysis of such cases diagnosed with histopathologic confirmation in our institute over the last seven years (2003-2009). In this study we reported 27 patients with zygomycosis from 61 cases with suspected mucormycosis from a single center (Hzt Rasool-e-Akram Hospital, a tertiary care center), with special reference to its prevalence, sites of involvement, underlying diseases, time of diagnosis and treatment strategy.

Higher prevalence rate $(29.6 \%)$ was observed in 2009. Rhino-orbito-cerebral type (100\%) was the only presentation which can be categorized as nasal-paranasal sinuses (77.8\%), orbital (11.1\%) and nasal-paranasal sinuses-orbital (11.1\%) involvement. Diabetes mellitus (in $55.7 \%$ of cases) was the most common underlying condition followed by hematologic malignancy (22.2\%). Twenty-six cases treated with combination of aggressive surgical debridement of necrotic tissue and amphotericin-B except for one case treated with amphotericin-B alone. Medical therapy included conventional amphotericin $\mathrm{B}(\mathrm{CAB})$ in most cases and in one patient liposomal amphotericin $B$ was used. In this study, onset of symptoms occurred in the summer and autumn in 21 out of 27 patients,

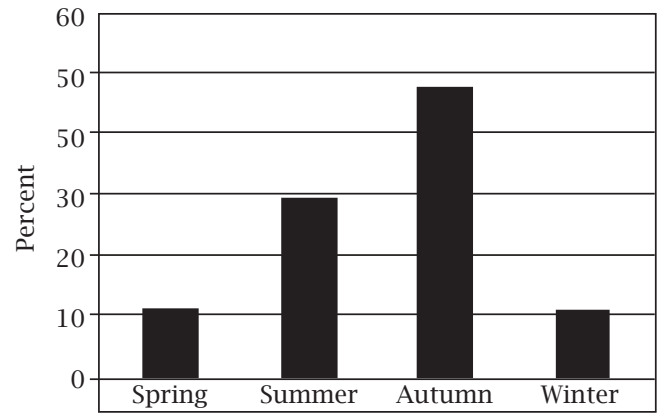

Figure 1: Seasonal distribution of zygomycosis cases.

showing a significant seasonal pattern in Iran $(\mathrm{p}=0.001)$, as had been reported in Lebanon. In Beirut, weather pattern analysis revealed clustering of onset of invasive mucormycosis at the end of a dry, warm period, which begins around May and ends in October, ${ }^{4}$ which was similar to the time of occurring symptoms in our patients (Figure 1).

The study highlights the importance of increased awareness for early diagnosis of zygomycosis and aggressive management. $\mathrm{Mu}-$ cormycosis incidence appears to be seasonal in Iran. This disease and its treatments are still associated with severe morbidity, disfigurement and disability.

\section{REFERENCES}

1. Chakrabarti A, Das A, Sharma A et al. Ten years' experience in zygomycosis at a tertiary care centre in India. J Infect 2001; 42:261-6.

2. Sims CR, Ostrosky-Zeichner L. Contemporary treatment and outcomes of zygomycosis in a non-oncologic tertiary care center. Arch of Med Research 2007; 38:90-3.

3. Roden MM, Zaoutis TE, Buchanan WL et al. Epidemiology and outcome of zygomycosis:a review of 929 reported cases. Clin Infect Dis 2005; 41:634-53.

4. Al-ajam MR, Bizri AR, Mokhbat J et al. Mucormycosis in the Eastern Mediterranean: a seasonal disease. Epidemiol Infect 2006; 134(2):341-6. 